\title{
BLOCKCHAIN and IoT for Enhancing Supply Chain Security - A review
}

\author{
Mir Mohammed Junaid Basha \\ Student \\ Industrial Eng. \& Mgmt. \\ RVCE, Bengaluru, India \\ Deepthi Galagali \\ Student \\ Industrial Eng. \& Mgmt. \\ RVCE, Bengaluru, India
}

\author{
Nikitha A P \\ Student \\ Industrial Eng. \& Mgmt. \\ RVCE, Bengaluru, India \\ Dr. C K Nagendra Gupta \\ Associate Professor and HOD \\ Industrial Eng. \& Mgmt. \\ RVCE, Bengaluru, India
}

\author{
Aiman Fathima K \\ Student \\ Industrial Eng. \& Mgmt. \\ RVCE, Bengaluru, India \\ Dr. K N Subramanya \\ Professor and Principal \\ Industrial Eng. \& Mgmt. \\ RVCE, Bengaluru, India
}

\begin{abstract}
In today's competitive business environment, the goal of a business is long-term survival. As seen that these days, most of the companies are customer driven. They mostly work on a combination of pull and push system Supply chain is an integral part of the manufacturing companies and various other sectors. Keeping this in mind, it is also found that the supply chain faces a lot of threats. This paper looks at various challenges a traditional supply chain faces such as Inventory theft, Information theft, Smuggling and Piracy, Trusting data to third party vendor. So, there is a high sense of need of security and monitoring for the data as well as the products. In this paper we discuss about the steps involved in implementation of Blockchain. This paper will summarize on how Blockchain, which on combining with IoT platform, will provide a real time and a secure supply chain. By doing so, the productivity, efficiency, and profitability of the supply chain can be improved.
\end{abstract}

Keywords:- Blockchain, Internet of Things (IoT), Supply chain, Blockchain of Things (BIoT), Security.

\section{INTRODUCTION}

As supply chains become more dynamic, include a range of stakeholders, and heavily rely on a variety of external counterparties. Blockchain has emerged as a viable candidate to de-tangle all data/documents/communication transactions that exist within the supply chain network. Every supply chain will have massive amount of data/ Information being exchanged between various stages in a supply chain. In order to handle huge of amount of data and ensure its security, supply chain can incorporate IoT and blockchain. This will help in improving security, productivity, efficiency, and profitability of the supply chain.

The IoT refers to the integration between connected devices for data collection and sensible decision taking. A lack of intrinsic protection safeguards however makes IoT exposed to threats to security and privacy. Blockchain can help to alleviate major security prerequisites in IoT with its Safety by Design. Blockchain features such as immutability, openness, traceability, data encryption and organizational flexibility can help overcome several of IoT 's architectural deficiencies. IoT would make it possible to track the technical condition of the equipment and the health and security of the workers. It can also provide additional communication instructions to all parties in the supply chain and improve in-transit visibility. ${ }^{1}$

While blockchain and the IoT solutions are powerful, businesses can exploit the combination of this advanced technology which is also referred to as Blockchain of Things (BIoT) in an incredible way. ${ }^{2}$ The study abbreviates on various security challenges supply chain faces due to massive amounts of data and incredibly enormous complexity. Information is often isolated, troublesome to access, formatted incoherently, or difficult to analyze. It might be overwhelming to think about driving innovation in any industry. Whereas the bright spot is that even the most basic applications of blockchain technology could massively improve productivity, efficiency, and profitability. The integrated incorporation of blockchain technology with IoT into the conventional supply chain network is illustrated in this report and demonstrates how various security issues can be handled.

\section{LITERATURE REVIEW}

Lei Xu, et $\boldsymbol{a l}^{3}{ }^{3}$ state that the current efforts of blockchain technology focus on transport data. The authors suggest a linking scheme using a new framework for handling the digital identity. The research identifies critical maritime transport challenges that could lead to incorrect information. Based on the result, it proposes a digital identification framework and a comprehensive design that can be incorporated with maritime supply chain management systems from Blockchain to reduce the incoherence of details. In addition, it proposes a scheme that helps bind the physical and cyber worlds and prevent cargo losses and reduce the burden of inspecting cargo.

Vishal Naidu, et al. ${ }^{4}$ presents an important approach for the automation and decentralization of supply chain management processes. The proposed system would make the whole tree of the supply chain a system in which each entity is part of the system. The system proposed provides an efficient and beneficial replacement for existing supply 
chain management systems. Using this system, the authors were able to reduce the error rates at different stages of the supply chain and improve customer support on a large scale. It would allow the supply chain tree to be completely retraced and traversed, helping to detect inconsistencies across different tree levels. This system ensures that current supply chain management systems are an effective and competitive substitute.

Mitsuaki Nakasumi ${ }^{5}$ examines blockchain-based solutions to address some of the supply chain problems such as Double Marginalization and Information Asymmetry etc. This research addresses the importance of building efficient supply chain. To develop it, the study says knowledge is one of the most important tools for producers in the supply chains. Because of the enormous amount of data generated and exchanged that is needed for the production activities, the paper specifies that it is important to recognize the most valuable ones and concentrate only on the "strategic transaction" leading to future changes at the supply chain level. The author discussed incentive problems related to major risk imbalances, such as risk of capacity. The effect of capacity risk is more severe for the decentralized supply chain because of the imbalance than for the vertically integrated supply chain. The author has suggested a blockchain-based approach to this problem to address the issue of double marginalization.

Lai Jieyu $\boldsymbol{u}^{\mathbf{6}}$ studies and analyzes, using block chain technology, the management of the logistics supply chain for e-commerce across borders. Starting with the main interpretation of the "decentralization" of the blockchain, this paper proposes its innovative application in three aspects of logistics, capital flow and information flow. The paper shows that, in China, e-commerce has made rapid progress across borders. It concludes that China's crossborder logistics supply chain will enter a new development period with the application of blockchain technology.

Chan Hyeok Lee, et al. $^{7}$ talk about the block chain IOT environment. When data is put into the block chain, this data can be leaked through a process called proof of work. The data can also be leaked through address search. So the study used a method called the knowledge zero proof to protect this. In this paper they explain data protection using smart contract and zero knowledge proof. Some third party can manipulate or retrieve the IOT data that is stored in a blockchain. The smart meter is installed for recording the device's power consumption and output. Security vulnerabilities have some privacy concerns for smart meters. So this paper says that the data can be kept safer and transactions can still be made using smart contract and zero knowledge.

Mohamed Awwad, et al. ${ }^{6}$ discuss how using blockchain technology can make supply chain management more efficient. Blockchain is a decentralized, digitized, and public ledger of all transactions in cryptocurrency. This can be used to combat counterfeit goods and to defend the brands. E-commerce, food, and warehousing are e- commerce industries that need more transparent and efficient supply chain. By using this technology, the transparency, risk reduction, flexibility, and speed can be enhanced as the process starts from customer's demand and ends with customer's delivery. The paper discusses the early adoption of block technology with IoT with specific emphasis on the degree of deployment of blockchain technology for purposes of validation, transparency and traceability in different industries.

From a detailed analysis of the papers' literature review, it can be concluded that the traditional centralized supply chain management model will slowly hamper the potential growth of e-cross-border commerce. The problems that arise are mainly due to the distance and location of the sites, making it difficult to coordinate, cooperate and monitor the flow. Hence, Blockchain technology can therefore be used as one of the solutions to the problem. It can effectively document transactions between the two parties in a verifiable and permanent manner. Ensuring that our data, files, or capital can be securely transferred is one of the strongest defenses. The current efforts of blockchain technology focus on transport data. IoT can be integrated with blockchain which is a system that operates over a network of uniquely operation techniques. The program makes sure the current supply chain management systems are an effective and competitive substitute.

This study is going to look at how the traditional supply chain functions can be secured upon implementation of IoT and Blockchain technology, which can furtur help solve security issues in traditonal supply chain.

\section{$>$ Supply Chain Processes}

The conventional supply chain process involves the procurement of raw materials and the creation of a tangible product which is passed to the customer afterwards. The supply chain comprises manufacturers, dealers , distributors, logistics firms, distribution centers and retailers. This is the network between a firm and its suppliers to manufacture and sell to the final customer a specific commodity. This network comprises a variety of activities, individuals, entities, information, and resources. Even the supply chain represents the steps it takes to distribute the product or service from its original location to the customer. A number of steps are involved in bringing consumers a product. It involves collecting and transforming raw materials into finished goods, shipping those products to end consumers and selling them there.

Information is important for the supply chain performance, as it serves as the framework on which the supply chain processes conduct transactions and managers make decisions. Certainly the need for exchanging knowledge through the different stages of the supply chain is of utmost importance. Information acts as a link between the various stages of the supply chain. It helps them to organize their acts, which offer many of the advantages of optimizing the supply chain's overall productivity. Clear information helps allow better use of the assets in the 
supply chain. It also helps in improving the co-ordination of supply chain flows in order to and reduce costs increase responsiveness. Without proper information, a manager will not know what customers require, how much inventory is left in stock, and when more additional products should be purchased, produced or shipped. Here are the few challenges that the organization may face due to a poor flow of information.
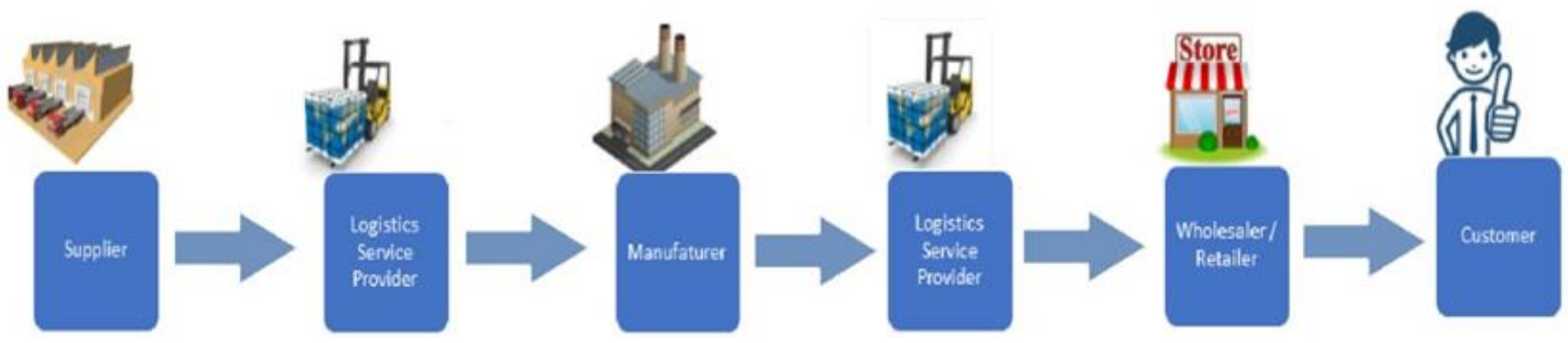

Fig 1:- Supply Chain Flow

The following are some of the problems facing modern supply chain ${ }^{7}$ :

- The lack of transparency from one end to the other causes a wide range of issues, such as theft, breach of code and conduct, etc.

- Lack of flexibility to respond to unforeseen changes in demand and operating cost regulation: demand is always changed with the impact of globalization.This results in operating costs getting increased indirectly.

- Many procedures involved in supply chain management are usually carried out manually, making them timeconsuming

- Inefficient supply chain risk management: an inefficient supply chain risk management system can not anticipate hazard and therefore can not respond to the situation.

- Lack of advanced technology: Modern supply chain management doesn't have an innovative technology to overcome the problem that emerged due to rapid changes due to globalization.

- Lack of real-time monitoring of the system, monitoring of the movement of products, the position of products within the storage, the shipment requests, and payment processing, etc.

\section{Supply Chain with IoT}

A conventional supply chain model can be briefly summarized as networking between all individuals, organizations, and activities engaged in the business process, from the supplier to the end consumer. Overseeing supply chains is a vital business feature for sectors such as retail, manufacturing, logistics, and e-commerce. Yet supply chain management can become increasingly complex due to growing customer demand, volatile market dynamics, and unforeseen delays. Besides, many procedures involved in supply chain management are usually carried out manually, making them timeconsuming. Corporate leaders are searching for ways that can make supply chain management more efficient, integrated, and effective.

However, as seen in the earlier section, there are several problems with conventional supply chain practices because of which the supply chain faces a wide range of challenges. Some of them are- no real-time monitoring of the system, no monitoring of the movement of products, the position of products within the storage, the shipment requests, and payment processing, etc which are very complex. Implementation of the IoT will solve these problems.

Role of Internet of Things in Supply Chain - IoT is a collection of interconnected physical devices capable of monitoring, recording, and transmitting and exchanging data. IoT devices are normally connected to networked computers through data or Wi-Fi networks. IoT devices employ equipment to determine various aspects of the world around them, such as location, temperature, humidity, light level, mobility, ability to handle, speed of processes and activities, and other environmental factors. IoT products come in a wide variety of different forms such as RFID processors, mobile devices, and portable sensors.

The IoT Systems can track, validate, and confirm goods and shipments in the supply chain by using GPS as well as many other technologies. The storage conditions of equipment can also be monitored using IoT devices to improve quality control across the supply chain. IoT is not a standalone technology, it is a mix of several technologies that will work to establish smartness. Other innovations include communications technology, information technology, electronic sensor, and actuator technology, and emerging advances in computing and analytics. 
ISSN No:-2456-2165

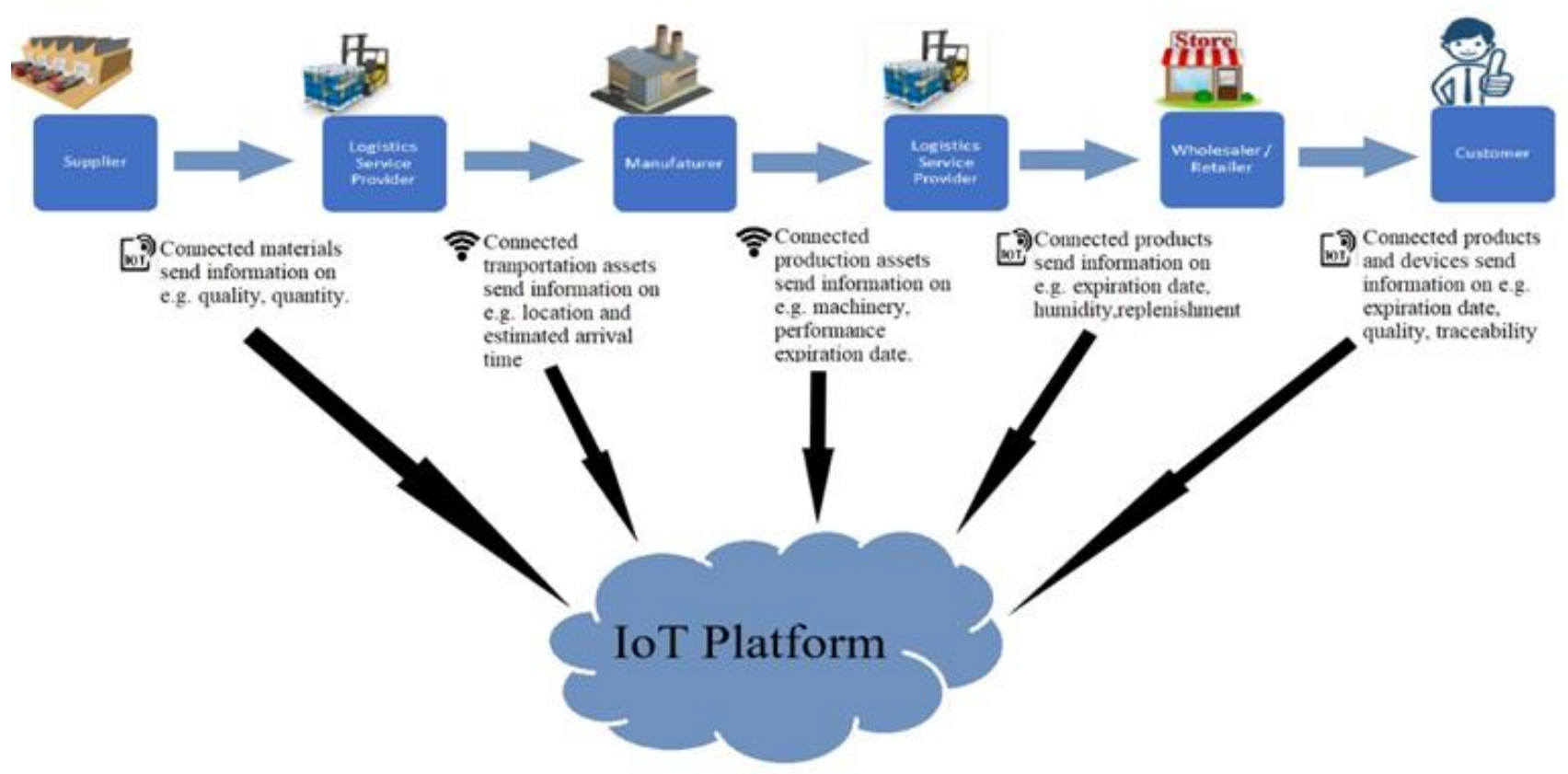

Fig 2:- Supply Chain with IoT Platform

Some of the benefits the supply chain can reap by the implementation of IoT are ${ }^{8}$ :

- Data Acquisition -IoT sensors may be mounted in a variety of items to understand how each consumer uses them. Using this technology, the patterns of use of various consumer populations can be analyzed and the data collected can be used when designing new goods.

- Monitoring the Products and Equipment - Businesses can agree on the threshold value of goods requiring defined temperature and humidity levels, e.g. food products. If the threshold limit is exceeded, IoT sensors can alert the concerned parties in the warehouse.

- Product Lifecycle Management - Material lifecycle management is also one of the main supply chain management practices. The growing batch of goods passes through stakeholders, such as suppliers, producers, B2B partners, mega stockists, wholesalers, and retailers. IoT sensors can be used to monitor goods across different supply chain systems to resolve issues such as under \& over-stocking. IoT technology may also help to find suppliers that are geologically appropriate for the industry.

- Management of Inventory - Businesses can mount IoT sensors in their inventory to provide real-time alerts on their stock rates. Information sent by IoT sensors will enable business leaders to evaluate the demand for different products.

- Logistics - Supply chain is made up of various fleet elements, such as containers, ships, and trucks, which are used to deliver products and consignments. By attaching these components to IoT, companies will have greater control over their delivery vehicles due to realtime tracking of vehicles.

- Maintenance of the facilities - The use of IoT in supply chain management will allow corporations to perform routine maintenance of all their equipment and delivery vehicles. IoT sensors capture real-time information from different cars, equipment, goods, supermarket shelves, and shop floors. chain:

Some of the disadvantages of utilising IoT in supply

Some of the IoT challenges apply to security and privacy problems. Apart from these, few other obstacles include interoperability, lack of regulations, legal challenges, rights issues, evolving issues in the IoT economy and other growth issues.

- Security of the information or the data is a key issue for any Web-connected industrial network. In a world where internet attacks have become more common, IoT technology may be another draw for hackers. Data processing and storage vulnerabilities can result in external attacks and leaks, the credibility of the business being recycled, and the cost of failure increased.

- Big data pools are one of the many benefits that the IoT offers to supply chain managers. However, with that power, it is the responsibility to obtain enough server power to store and process all the data collected.

- IoT relies heavily on getting a secure Internet connection. Because fleet drivers are traveling from one place to another, there is not always a secure network. Internet speed is yet another thing to be considered while integrating IoT into the supply chain.

- Supply chains also span thousands of kilometres throughout the globe. Problems with integration in IoT implementations can also prove very difficult to solve.

- Having a team that is knowledgeable enough to develop a solution that caters to an organization is a challenge on its own, despite the lack of specialized IoT training.

- Illustrating the security policies and setting up guidelines for the use of corporate networks is a timeconsuming procedure. 


\section{Supply Chain with BLOCKCHAIN}

The study has shown how the conventional supply chain of a company is, and how IoT technology can be used to make it faster and more efficient. It is seen that as the use of IoT increases, the possibility to misuse also increases. However, by integrating blockchain technology into its architecture, these risks could be mitigated.
Blockchain is a detailed data archive where all communications between IoT devices is stored in cloud. This provides instant access to all product related information. In this manner both the company and the consumer can use blockchain and IoT technologies to track the entire product life cycle over the entire supply chain.

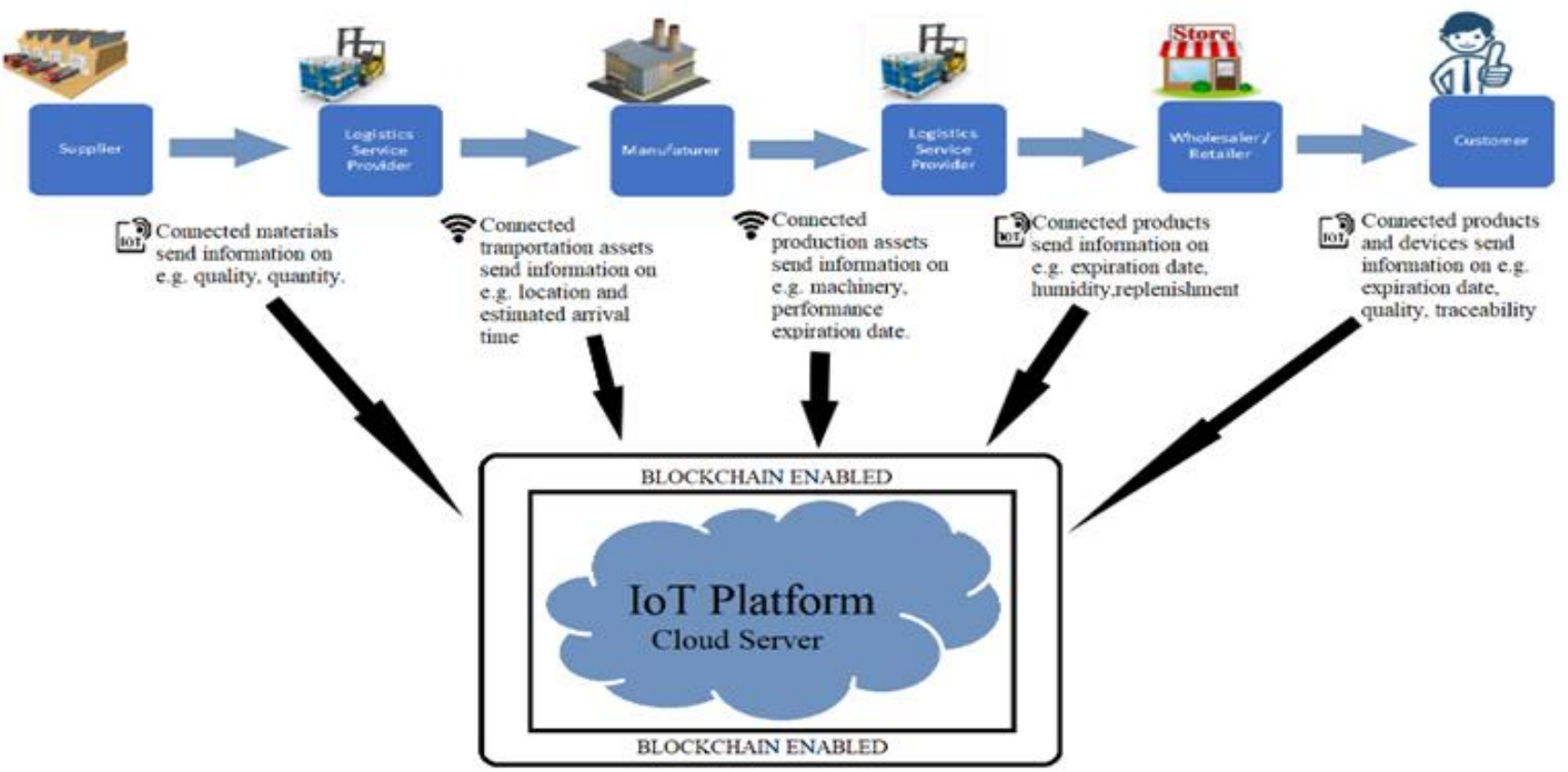

Fig 3:- Supply Chain with IoT Enabled Blockchain

IoT serves as a link between the physical and the digital world by providing reliable information on the products and materials in a supply chain. By having IoT enabled with Blockchain in the Supply Chain, there is easier access to information, linking both physical and information flows and making it more secure, reliable, transparent, and cost-effective.

\section{Implementation of Blockchain ${ }^{4}$ -}

STEP 1: Set up a private blockchain network Ethereum can be used to set up a private blockchain network. It is a blockchain-based open source distributed computing network. Each entity must set up a high computational power machine, known as the Ethereum node, to create an Ethereum block chain. Applications that are available on different platforms such as GoEthereum or 'geth' can also be used to create an Ethereum node.

STEP 2: Create Smart Contracts - Ethereum's more sophisticated feature is Smart Contract. It's a software that makes money, materials, properties, shares or anything of value easier to exchange. The smart contract is a computer program that works independently and automatically when those requirements are met. Which works exactly as planned, without any risk of third parties interrupting, censoring, corrupting or interfering.
Like in conventional intranet systems, where there is a centralized repository and a centralized database containing all the data on the supply chain processes that the organization handles; Smart Contracts is a single address floating database for all nodes, and this address is a contact point for the network. A smart contract can be written in programming languages such as JavaScript, C++. Online compilers are available for these programming languages, which can be used to write smart contracts. The contract can be published on a particular network of the Ethereum block chain.

STEP 3: Build Distributed Application - A Distributed Application is a software program designed primarily for gathering or uploading data from or into a floating Smart Contract Ledger. This Smart Contract ledger is mostly hosted on cloud storage platforms and operates concurrently on multiple systems. A simple approach to creating this distributed blockchain application is to create a web page using HTML / CSS and then use an API called Web3Js, the interface to the block chain of Ethereum. 


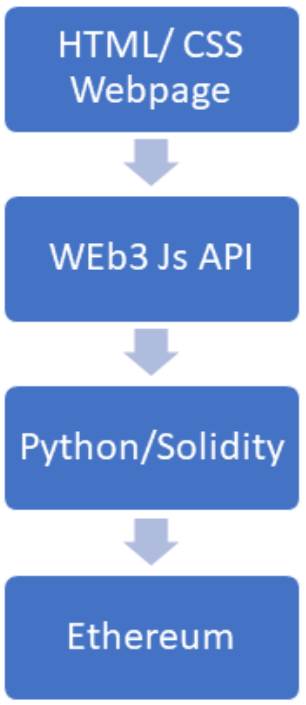

Fig 4:- Ethereum Block Chain

Figure 4 shows the development stack of the distributed application in the Ethereum block chain. The last 3 layers of the stack must remain unchanged in the Ethereum network. It may be replaced by any other scripting language for a distributed application to be rendered on a web page other than HTML / CSS, provided that it has Web3J support. chain:

Advantage of using Blockchain enabled IoT in Supply

There are numerous problems associated with the traditional supply chain structure, but there is interest in how blockchains can help secure the supply chain industry given the complexity and lack of accountability of our existing supply chains.

The following are the few challenges of supply chain that blockchain could help protect ${ }^{9}$ :

$>$ Inventory Theft

$>$ Information Theft

$>$ Smuggling and Piracy

$>$ Trusting Data to Third Party Vendor

$>$ IoT Sensor Compromise

Blockchain methodology for addresing each of the concerns above.

- Inventory Theft - Theft of inventories by workers, particularly in distribution centers, remains a major threat to supply chains. The size of the goods passing through these centers makes it impossible to track everything precisely. Therefore, theft operations are often complex. It involves employees within the company working with people outside the company, such as drivers, to move stolen goods outside the distribution center for resale.

Here using IoT technology if each of the inventory is tagged, then the flow of the inventory can be observed remotely, and the inventory can be tracked with precision. These tags containing information of the inventory can be stored in blockchain further providing security to the information of the inventory, its intended purpose, destination any other sensitive data.

- Information Theft - Cloud data security is needed as supply chain technologies and data storage systems migrate to the cloud. Inability to manage cloud access properly will result in serious IT risks, including, or worse, giving users an excess of privileges. This will leave the cloud data open to anyone interested in accessing it.

This safety concern can also be tackled by using blockchain. It can be done by including strong permissions and access management. This way, the access to the cloud information can be secured. The information or data can be encrypted and stored, and the access keys can be randomized and easily be shared between the parties without any fear of hacking.

- Smuggling and Piracy - Smuggling goods is a potential risk to the security of the supply chain. Counterfeit or pirated goods, counterfeit products and so-called gray market products are smuggled across countries using shipping companies. This not only impacts the destination and customers, but also affects the supply chain by disrupting its smooth flow.

Using blockchain helps to monitor and track in every step. It records details such as where and when goods are in logistics. The blockchain feature ensures that nobody involved in the transaction can edit, modify or delete any record without the express approval of the rest. This is of key importance because it avoids the fraud in freight trade and the illegal smuggling of goods.

- Trusting Data to a Third-Party Vendor - Cloud service providers invest a tremendous amount of money to ensure that their websites, reputations, are safe. It is for the very reason that they rely on their livelihoods. Several companies are still, understandably, unwilling to entrust sensitive data to third party vendors. Using a cloud platform that does not put a security premium to increase the risk. It is also important to hire only those companies with experience in managing sensitive applications and to pass strict safety audits. Such organizations also have much higher requirements and more security layers than the organizations whose data they host.

Trusting data to a third party is very risky. Yet many companies who cannot afford to allocate funds to safeguard their own data, rely on third party vendors. The level of security can be compromised when transferring the data or when accessing the data from a third-party vendor. Here, blockchain can help ease the flow of information by maintaining the highest level of security. By doing so, the information is transferred in blocks. It is these blocks that are encrypted making it highly secure. 
- IoT Sensor Compromise-IoT devices are those that have connected sensors to the internet. They are increasingly being used to manage inventories in supply chains. They are also used before occurrence to predict machine failures. However, each of these sensor data is another tool that hackers can use to gather supply chain information, including order volumes, key supplier relationships, and more. IoT devices should be tested and reviewed for protection and encryption at all points in the IoT environment, and this blockchain technology is used for this purpose. The information can be stored in encryption within the IoT devices, which practically makes it impossible for hackers to gather or retrieve any information from it.

\section{CONCLUSION}

It is seen that supply chain faces huge obstacles and will face more tomorrow. The combination of blockchain and IoT technologies would help the supply chain to reduce its risks. The use of the block chain will ensure the authenticity of the information. Thus, preventing possible fraud and making the information available in an easy and secure format. On the other hand, by providing accurate information on supply chain materials and goods, Iot can act as a bridge between the physical and digital worlds. The combination of these two will therefore provide a safer option for the overall protection of the supply chain.

\section{REFERENCES}

[1]. Panarello, A., Tapas, N., Merlino, G., Longo, F., \& Puliafito, A. (2018). Blockchain and iot integration: A systematic survey. Sensors, 18(8), 2575.

[2]. Lerner, S. (n.d.). Enterprise Mobility Exchange. 5 Revolutionary Advantages of Combining Blockchain With Internet of Things | Enterprise Mobility Exchange. Retrieved from http://www.enterprisemobilityexchange.com/emecollaboration-tools/news/5-revolutionary-advantagesof-combining-blockchain-with-internet-of-things

[3]. Lei Xu, Lin Chen, Zhimin Gao, and Yanling Chang, Eleftherios Iakovou and Weidong Shi "Binding the Physical and Cyber Worlds: A Blockchain Approach for Cargo Supply Chain Security Enhancement”, 2018 IEEE International Symposium on Technologies for Homeland Security (HST)

[4]. Vishal Naidu, Kumaresan Mudliar, and Abhishek Naik, Prof Prasenjit Bhavathankar “. A Fully Observable Supply Chain Management System Using Block Chain and IOT", 2018 3rd International Conference for Convergence in Technology (I2CT)

[5]. Mitsuaki Nakasumi "Information Sharing for Supply Chain Management based on Block Chain Technology", 2017 IEEE 19th Conference on Business Informatics
[6]. Lai Jieyu "Research on Cross-border E-commerce Logistics Supply under Block Chain", 2019 International Conference on Computer Network, Electronic and Automation (ICCNEA)

[7]. Satyabrata Aich, Sabyasachi Chakraborty, Mangal Sain, Hye-in Lee, Hee-Cheol Kim "A Review on Benefits of IoT Integrated Blockchain based Supply Chain Management Implementations across Different Sectors with Case Study", International Conference on Advanced Communications Technology (ICACT)

[8]. Joshi, N. (n.d.). Application development | Big data| IoT | Digital Business | Cloud. IoT in supply chain management | Artificial Intelligence | Retrieved from http://www.allerin.com/blog/building-smarter-supplychains-with-iot.

[9]. Wainstein, L. (n.d.). The Network Effect - Beyond Supply Chains. 7 Supply Chain Security Concerns to Address in 2019 - The Network Effect. Retrieved from http://supplychainbeyond.com/7-supply-chainsecurity-concerns-to-address-in-2019/

[10]. Chan Hyeok Lee, Ki-Hyung "Implementation of IoT System using BlockChain with Authentication and Data Protection”, 2018 International Conference on Information Networking (ICOIN)

[11]. Mohamed Awwad, Sohit Reddy Kalluru, Varun Kazhana Airpulli, Madhubala Santosh Zambre, Aniket Marathe and Prasham Jain "Blockchain Technology for Efficient Management of Supply Chain", Proceedings of the International Conference on Industrial

[12]. Marwa Chamekh, Mohamed Hamdi, Sadok El Asmi, and Tai-Hoon Kim "Secured Distributed IoT Based Supply Chain Architecture", 2018 IEEE 27 $7^{\text {th }}$ International Conference on Enabling Technologies: Infrastructure for Collaborative Enterprises

[13]. Abderahman Rejeb, John G. Keogh, and Horst Treiblmaier "Leveraging the Internet of Things and Blockchain Technology in Supply Chain Management", Future Internet, Vol.11, No. 7, pp. 122.

[14]. Amal Alahmadi, Xiaodong Lin "Towards Secure and Fair IIoT-Enabled Supply Chain Management via Blockchain-based Smart Contracts”, ICC 2019 - 2019 IEEE International Conference on Communications (ICC)

[15]. Pavan Manjunath, Rajashree Soman,and Dr. Pritam Gajkumar Shah "IoT and Block Chain driven Intelligent Transportation System", 2018 Second International Conference on Green Computing and Internet of Things (ICGCIoT)

[16]. Engineering and Operations Management Washington DC, USA, 2018 